\title{
Design and Realization of Open CNC System Based on Software Motion Controller
}

\author{
Xu Ran ${ }^{1, ~ a, ~ J i a n q u n ~ L i u ̈, ~ b, ~ W e i q i a n g ~ G a o ~}{ }^{3, c}$ and Guoqiang Huang ${ }^{4, d}$ \\ ${ }^{1-4}$ Guangdong University of Technology, Guangzhou, China \\ aclarencerex@foxmail.com, bijliu@sina.com, wogalt@139.com, ${ }^{\text {dh }}$ 'wuang_gq@qq.com
}

Keywords: A3200; CNC system; Laser interferometer; Error calibration

Abstract. The open CNC system based on $A 3200$ has been designed and realized. Firstly, the hardware platform design of the system was introduced in the paper. Then, the HMI(Human Machine Interface) of the system has been introduced,which was developed by $Q t 4.8$. Afterwards, the process of the positional accuracy measurement and error calibration of the system has been illustrated. Finally, the system has met the requirement of real-time motion control and positional accuracy, and it has worked well.

\section{Introduction}

An open CNC system is built on an open platform, realizing the open ability, interchangeability, transportability and extendibility, and it has a modular structure ${ }^{[1,2]} . A 3200$ is a software based motion controller that uses FireWire(IEEE-1394) to provide up to 32 axes of coordinated motion, networked with intelligent drives ${ }^{[3]}$. It allows the user to choose the controlling mode and the numbers of servo axes based on diffrent configure to meet the practical requirement of an open CNC system. $Q t$ is a development framework of application and user interface. It makes it possible that the software developed by $Q t$ can run on different operating system without repeatedly compiling source codes ${ }^{[4]}$.

The open CNC system has taken "IPC(Industrial Personal Computer) \& SMC(Software based Motion Controller)" as the structure of the system, and it is a full-closed loop system. The software system running under windows operating system has been developed with Qt4.8 based on the object-oriented program design and the module techniques. After installation, configuring, measurement and calibration, the system has met the design requirement.

\section{Design of the Hardware Platform}

\begin{tabular}{|c|}
\hline IPC(industrial personal computer) \\
\hline Windows OS \& INtime \& HMI \& A3200 \\
\hline IEEE 1394 firewire card \\
\hline
\end{tabular}

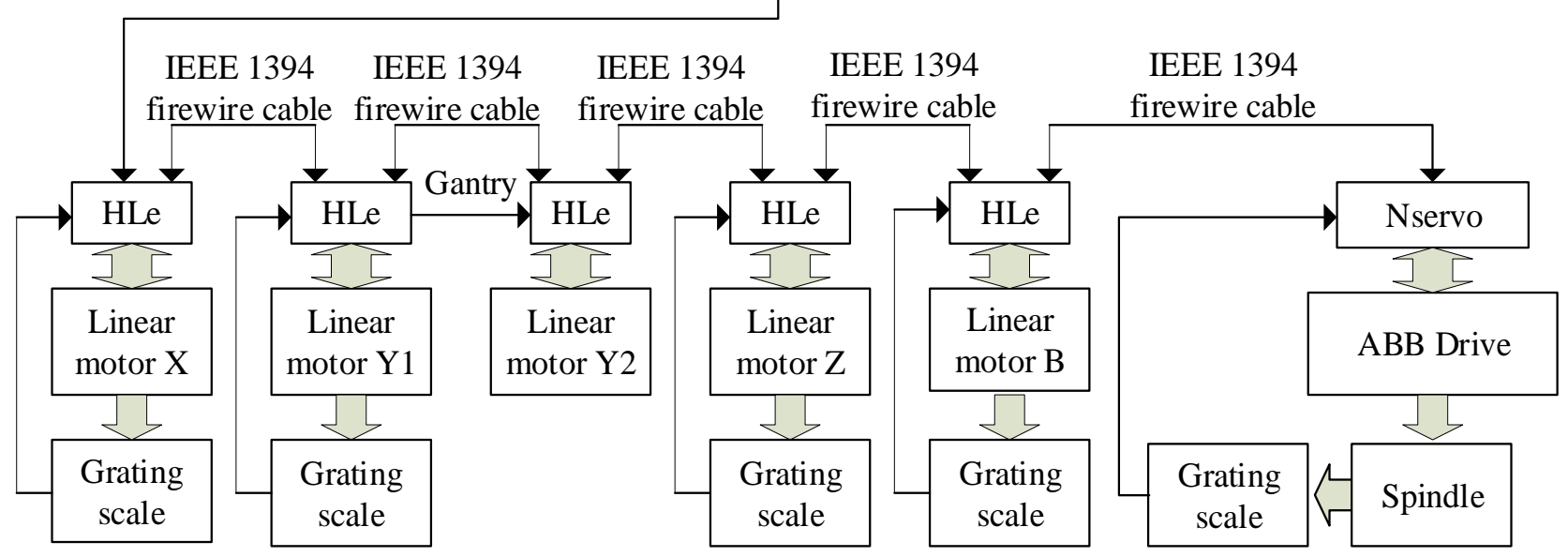

Fig. 1. Hardware structure of the system 
The system has been made up of software platform and hardware platform. It was the IPC that the connection between software platform and hardware platform. The hardware platform has been composed of Ndrive HLe digital drive, BLMX Series linear motor, Heidenhain grating scale, Nservo, Air bearing spindle and so on. The hardware structure of the system is shown in Fig.1. The machine tool platform and the electric control cabinet are shown in Fig.2 and Fig.3.

Ndrive HLe digital drive is a high performance linear amplifier. The communication transmission rate is up to $3.2 \mathrm{Gbps}$ with 1394 firewire so that it makes certain the feature of real-time motion control. BLMX Series linear motors with nonmagnetic forcer eliminates cogging and magnetic attraction to allow for extremely smooth motion, very tight velocity and position control, which are ideal for applications demanding the ultimate in force output. LIP400 series of the Heidenhain grating scale is for meeting the high-precision measurement of the system.

The system has worked for the five-axes ultra-precision machine tool in our laboratory. According to the layout of the machine tool, the Y-axis has taken the gantry mode, which has used the force from air cylinder to balance the weight of the principal axis. With the Nservo, the running mode of the spindle can be switched between principal axis mode and $\mathrm{C}$-axis mode.

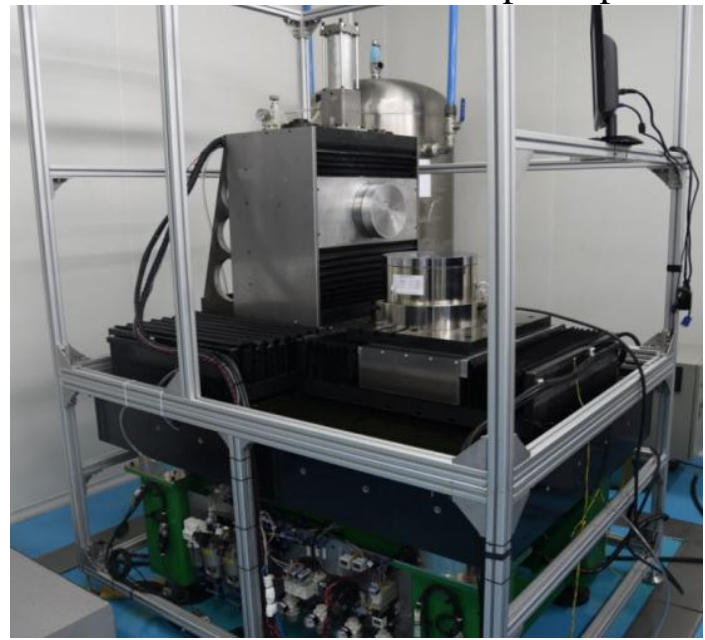

Fig. 2. Machine tool platform

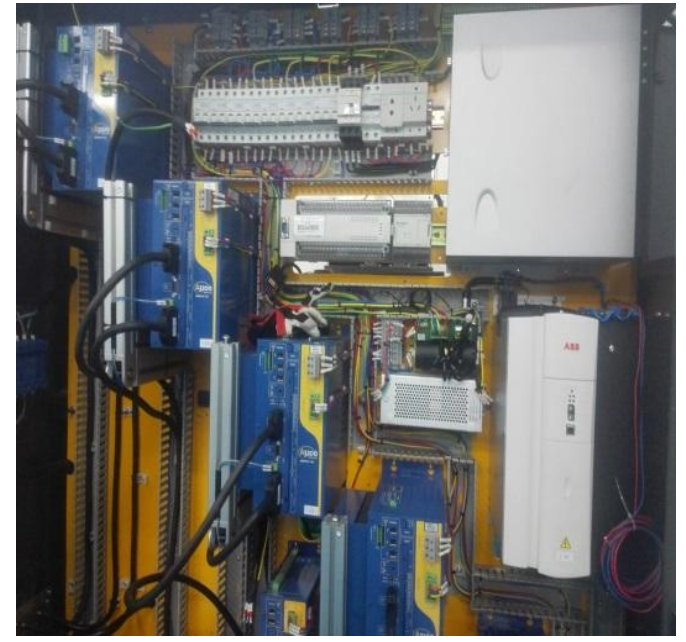

Fig. 3. Electric control cabinet

\section{Design of the Software Platform}

The software platform has been composed of windows operating system, INtime real-time extension, A3200 software controller and the HMI of the system. The HMI is responsible for the function of computer interaction and real-time display. A3200 deals with the real-time tasks, such as the interpolation calculating, position control, cutter compensation, velocity dealing and so on. A3200 runs under windows operating system in a real-time kernel that runs independently of other programs running on the IPC, which is responsible for the real-time motion control tasks and data sharing by the shared memory with the windows operating system ${ }^{[5]}$.

Function Module of the HMI. The motion control interface of the CNC system is shown in Fig.4. According to the design requirement, the application had following function modules:

1)Product: enabling, homing and motion controlling of single axis or multiple axes, loading and editing of NC program, display of running status and other assistant functions. There are four motion controlling mode to satisfy different application, such as Auto Mode, Handle Feed Mode, Incremental Mode, Home Mode. When the system is in one mode, some function buttons are unavailable for avoiding misoperation.

2)Edit: surface modeling of parts, post-processing and generating the NC program.

3)Utility: generating the test program of error measurement, simulation of machining process.

4)Diagnose: monitoring the running status of the open CNC system, diagnosing the fault of the system and finishing the restoration of the system.

5)Setup: managing the parameters of the open CNC system, workpiece, cutter, processing craft and setting the coordinated system. 


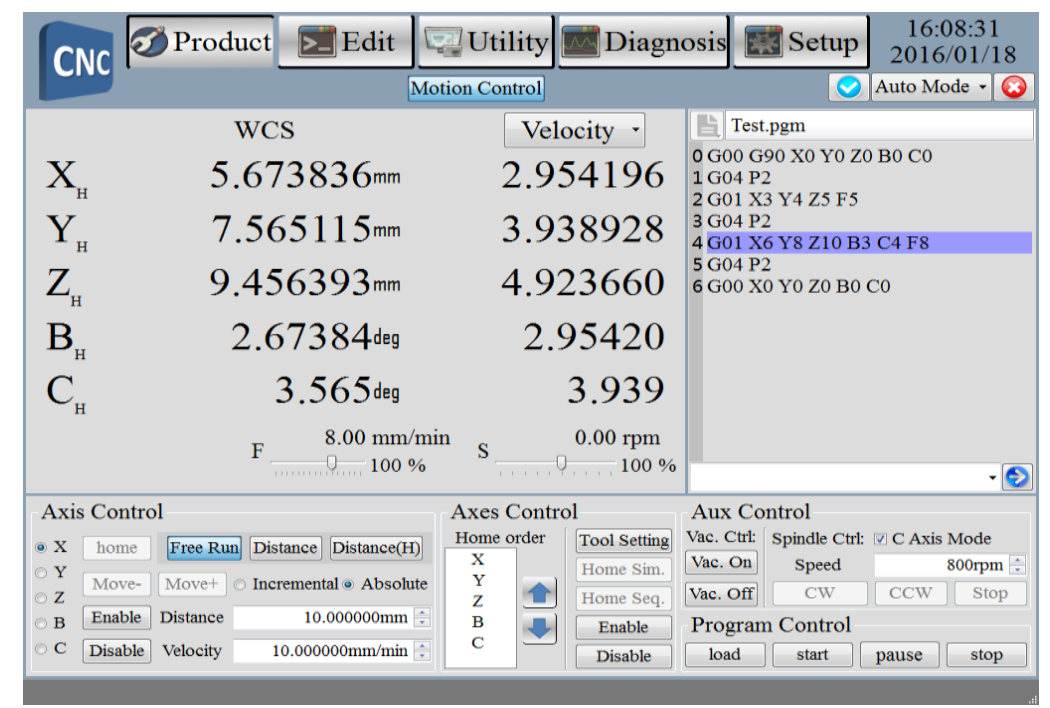

Fig. 4. Motion control interface of the CNC system

\section{Measurement of Positional Accuracy and Error Calibration}

Measurement of Positional Accuracy. Positional accuracy is an important performance to evaluate the CNC system, it affects the processing quality of the system. To meet the requirement of high velocity and high accuracy for the system, the positional error has been measured and calibrated.

According to the measurement principle of Dual-frequency Laser Interferometer ${ }^{[6]}$, the measurement of the linear positional accuracy has been accomplished with ReniShaw XL_80. The measurement and the calibration of $Z$-axis were used to illustrate the whole process.

Within the scope of travel(-85 +85mm), the 35 mark points selected randomly, $3 \mathrm{~mm}$ for the skip distance, $2 \mathrm{~mm} / \mathrm{s}$ for the feedrate, $4 \mathrm{~s}$ for dwell time and 5 double-direction measurements have been chosen to generate the test program. The dwell time made it sure that the Laser interferometer had enough time for collecting positional error data of the mark points. The results of measurements before and after calibration have been analyzed upon VDI/DGQ 3441 standard with the analysis software provided by Laser Interferometer. The result of the measurements before calibration is shown in Fig.5.

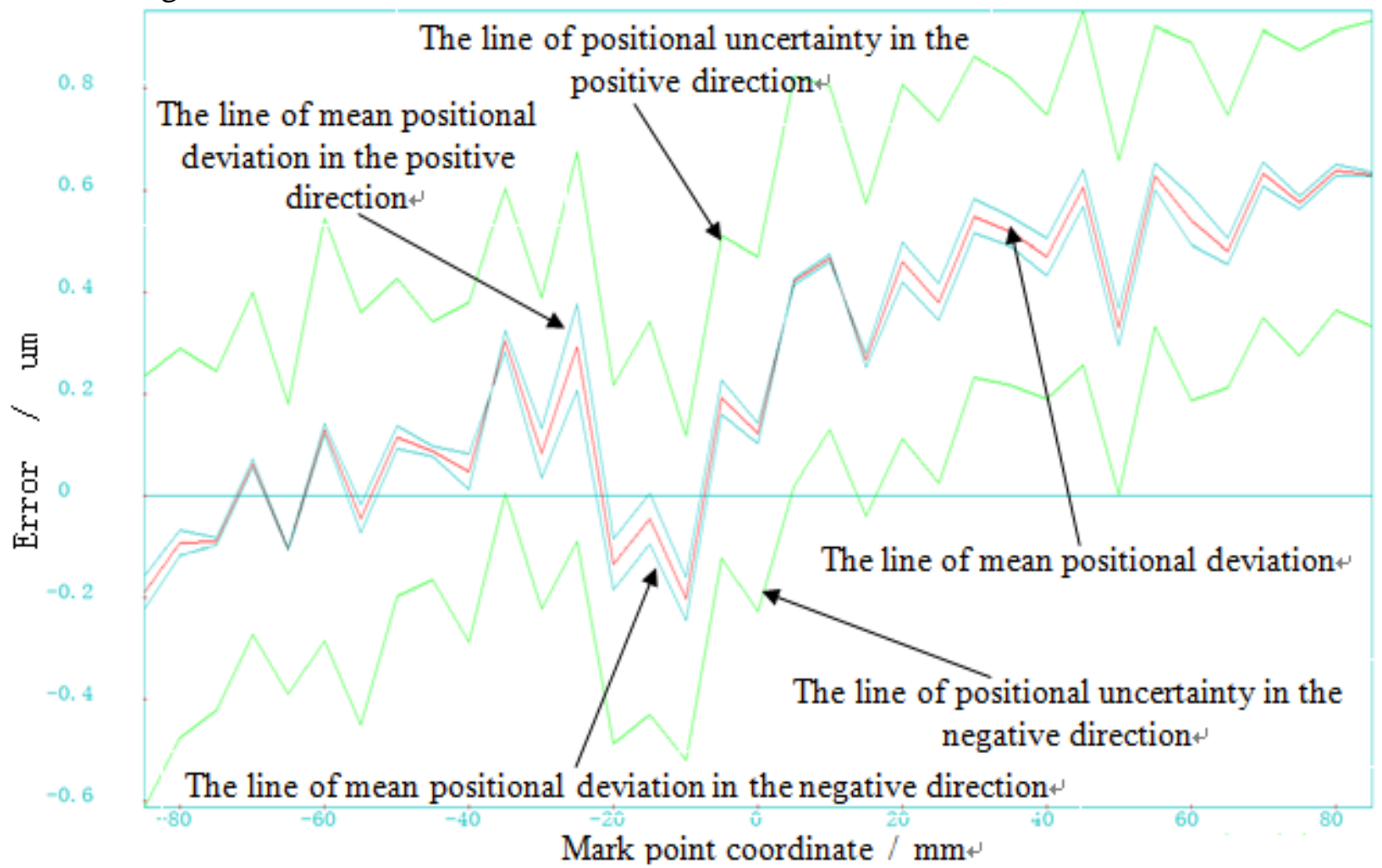

Fig. 5. Result of the measurements before calibration 
Error Calibration. There are two software error calibration methods for CNC system. One is the backlash calibration method, the other is the thread pitch error calibration method. The former has been taken to calibrate the error of the system.

1D or 2D error calibration file is accepted by A3200 configuration Manager provided by A3200. According to the error measured by Dual-frequency Laser Interferometer, it has been generated that the error calibration file with the format required by A3200, and it has been loaded to A3200 configuration Manager.

Within the scope of travel, the travel has been divided randomly for 35 mark points. The error of mark point has been measured by Dual-frequency Laser Interferometer. And the average value of double direction error has been calculated for $\bar{\varepsilon}_{i}$. With the calibration file, the average value has been considered by the system. The calibration value of the error at the mark point has been obtained as below:

$$
\varepsilon_{i}+\varepsilon_{i}^{\prime}=0 \quad(\mathrm{i}=1,2,3, \ldots, 35)
$$

where $\varepsilon_{i}$ : error of mark point; $\varepsilon_{i}^{\prime}$ calibration value of mark point.

In order to get better positional accuracy, more measurements of double direction movement have been taken, and the average of the error at the same mark point has been obtained as below:

$$
\overline{\varepsilon_{i}}=\frac{1}{n} \times \sum_{i=1}^{n} \varepsilon_{i}
$$

where $\overline{\varepsilon_{i}}$ : average of the error value at the same mark point; n: times of measuring the same mark point.

After calibrating error of mark points, the five double-direction measurements have been done again. The result of the measurements after calibration is shown in Fig.6. The positional accuracy and the repeated accuracy have been improved obviously according to the contrast between Fig.5 and Fig.6, which have been obtained after calibration. The contrast of analysis results before and after error calibration is shown in Table 1 . The system has met the requirement, which the positional accuracy was $1 \mathrm{um}$. It has been proved effective of the calibration method according to the results.

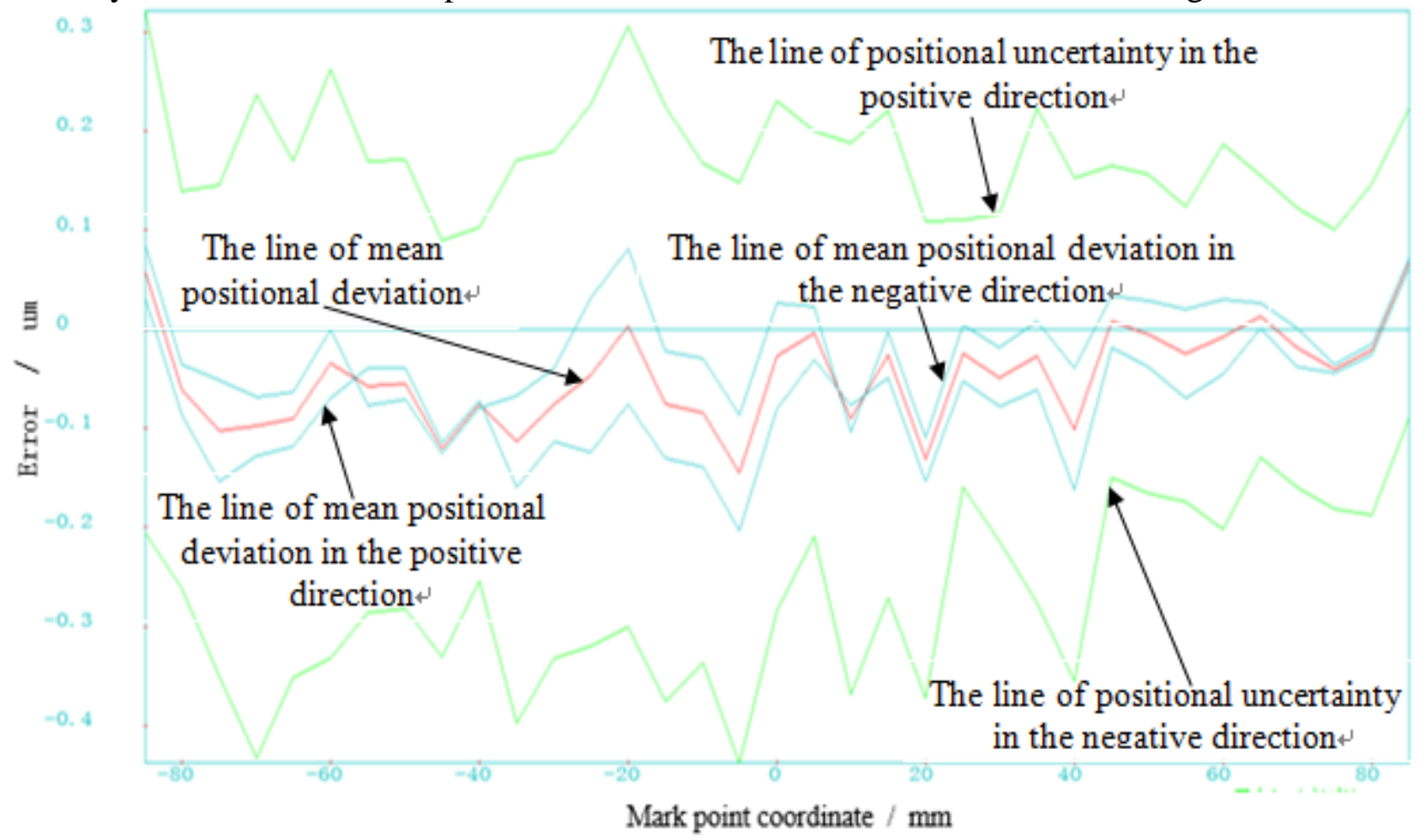

Fig. 6. Result of the measurements after calibration 
Table 1 Contrast of analysis results before and after error calibration

\begin{tabular}{|c|c|c|}
\hline Items & Before calibration & After calibration \\
\hline Positional uncertainty $(\mathrm{P})[\mathrm{um}]$ & 1.564 & 0.760 \\
\hline Maximum scatter $\left(\mathrm{P}_{\mathrm{s} \text { max }}\right)[\mathrm{um}]$ & 0.808 & 0.610 \\
\hline Maximum reversal $(\mathrm{U}$ max) $[\mathrm{um}]$ & 0.169 & 0.156 \\
\hline Positional deviation $(\mathrm{Pa})[\mathrm{um}]$ & 0.840 & 0.212 \\
\hline Mean reversal (U mean) [um] & 0.055 & 0.064 \\
\hline Mean scatter (Ps mean) [um] & 0.607 & 0.384 \\
\hline
\end{tabular}

\section{Conclusions}

The open CNC system based on A3200 has been accomplished, which has a good interaction interface. Through calibrating the positional error, the system has obtained the high positional accuracy and high repeated accuracy. The system has achieved $\mathrm{P}=0.76 \mathrm{um}$ and $\mathrm{P}_{\mathrm{s} \max }=0.61 \mathrm{um}$ in Z-axis. The open CNC system not only has provided more choices and flexibility for users but also has reduced the amount of the hardware to improve the stability and reliability. It has been proved that the open CNC system has met the design requirement of the system and worked well. Further research will focus on aspheric processing with the system.

\section{Acknowledgements}

This work was supported by the University Industry Science Partnership (UNISPAR) Project of Guangdong Province and Ministry of Education of P.R. China (No.2013B090500125, No.2013B090500037), the Science and Technology Planning Project of Guangdong Province (No.2015B010101013, No.2015B010102012), the Postgraduate Demonstration Base Project of Department of Education of Guangdong Province (No.2013JDXM29), and the Introduction of Innovative R\&D Team Program of Guangdong Province (No.201001G0104781202). Meanwhile, as the first author, I would like to express my gratitude to all those who have helped me during the writing of this thesis.

\section{References}

[1] Shucheng Zhang: Journal of Jiamusi University(Natural Science Edition), Vol.30 (2012) No.1, pp.83 85. (In Chinese)

[2] Shu Zhang, Liuqun Fan, Zhihao Zhu, Xu Jin: Manufacturing Technology \& Machine Tool, (2012) No.2, pp.9 12. (In Chinese)

[3] Information on http://www.aerotech.com.

[4] Yafei Huo, in: The Quick start of Qt Creator (second), edtied by Beihang University Press, Beijing, China(2014). (In Chinese)

[5] Information on http://www.intime.com.

[6] Jianhui Zhang: Machine Tool \& Hydraulics, Vol.39 (2011) No.4, pp.114 115. (In Chinese) 